\title{
Kernos
}

Revue internationale et pluridisciplinaire de religion grecque antique

$16 \mid 2003$

Varia

\section{Le regard rationnel de Platon sur les dieux traditionnels}

\author{
Aikaterini Lefka
}

\section{OpenEdition}

\section{Journals}

Édition électronique

URL : http://journals.openedition.org/kernos/814

DOI : $10.4000 /$ kernos.814

ISSN : 2034-7871

\section{Éditeur}

Centre international d'étude de la religion grecque antique

\section{Édition imprimée}

Date de publication : 1 janvier 2003

Pagination : 125-132

ISSN : 0776-3824

\section{Référence électronique}

Aikaterini Lefka, «Le regard rationnel de Platon sur les dieux traditionnels », Kernos [En ligne],

16 | 2003, mis en ligne le 14 avril 2011, consulté le 01 mai 2019. URL : http://journals.openedition.org/ kernos/814; DOI : 10.4000/kernos.814 


\section{Le regard rationnel de Platon sur les dieux traditionnels}

\section{Introduction}

Les premiers penseurs grecs ont porté sur les croyances religieuses de leur époque un regard très critique. Cette attitude s'intègre dans la remise en question globale de la version courante de la vérité sur le monde qui nous entoure. Xénophane ${ }^{1}$ et Héraclite $^{2}$, notamment, ont insisté sur le rejet des traits trop anthropomorphiques des dieux, tels qu'ils apparaissent dans la mythologie traditionnelle. Ces mythes étaient véhiculés dans toute la Grèce par les grands poèmes épiques d'Homère et d'Hésiode. Les premiers philosophes mettent en évidence la subjectivité et la relativité des croyances humaines; aussi se dressent-ils contre les dires arbitraires des poètes.

Platon, pour sa part, suit ce courant, à la manière qui lui est propre. Il insiste, par exemple, sur l'importance de l'adhésion religieuse aux divinités traditionnelles. D'ailleurs, il mentionne par leur nom propre pas moins de 76 divinités ou groupes de divinités, et ce à 1182 reprises. Or, ces divinités se trouvent subordonnées hiérarchiquement à leur « créateur et père », le Démiurge du Timée, aussi étonnant qu'original. La personnalité de chaque divinité apparaît également « remodelée », car Platon effectue d'une part une sélection des données des croyances courantes, selon des critères rationnels dont nous parlerons dans la suite, et, d'autre part, y ajoute de nouveaux éléments, notamment liés à l'exercice de l'intellect et de la dialectique ou à ses propres conceptions philosophiques. En effet, Platon est le premier penseur qui se propose d'introduire, par le biais de la rationalité humaine, des règles théologiques plus strictes dans le domaine de la religion grecque, laquelle se distingue par une grande diversité et une grande liberté de croyances.

La manière dont les dieux se présentent dans les dialogues platoniciens suit presque toujours ces «principes théologiques », que nous allons d'abord rappeler. Or, dans certains cas exceptionnels, nous constatons des écarts ou même des contradictions. Ce sont quelques exemples significatifs de ces mentions, qui n'ont pas encore retenu l'attention des chercheurs, dont nous allons nous préoccuper particulièrement, en proposant des interprétations possibles, afin de mieux définir la véritable portée des règles rationnelles que Platon s' impose à lui-même concernant la conception de la divinité . $^{2}$

1 Xénophane, $21 \mathrm{~B}$ 11-16 et 23-26 Diels-Kranz.

2 Héraclite, 22 B 5, B 14, B 15, B 32, B 67 Diels-Kranz.

3 Cet article est une version plus élaborée de la communication présentée au VIII Colloque International du C.I.E.R.G.A., «Religion et Rationalité en Grèce Ancienne " (Rhodes, 25-29 mai 2001). 


\section{Les typoi peri theologias}

Platon ne se contente pas d'adresser une critique véhémente aux auteurs de poèmes qui présentent les dieux de manière inacceptable. Il ressent le besoin de

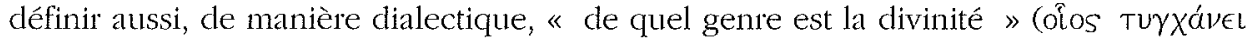

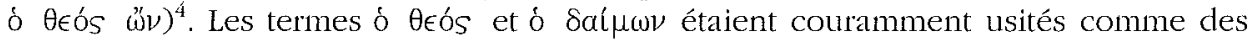
synonymes, autant au singulier qu'au pluriel, pour désigner « la divinité » ou « les dieux » en général (le terme $\delta a l \mu \omega \nu$ étant également utilisé plus spécifiquement pour des divinités mineures). Platon se propose de rechercher quel est le genre, ou l'є̇̊os de tout être qui peut être appelé « dieu ». Notons que le philosophe évite d'utiliser l'ős (qui) et se contente de l'ốos (de quel genre). Aussi garde-t-il sa distance par rapport à l'identité particulière de la divinité, certitude impossible pour les hommes, comme il le souligne aussi dans le Cratyle

Les termes tútrol $\pi \epsilon \rho l$ $\theta \epsilon o \lambda o \gamma\left(a s^{6}\right.$ utilisés par le philosophe pour désigner les traits caractéristiques du genre divin ont fait couler beaucoup d'encre savante et restent toujours controversés. D'après $\mathrm{R}$. Bodéüs, « le tupos est la matrice d'après laquelle on reproduit des exemplaires conformes. Le mot ici est propre à désigner le paradigme idéal». Selon cet auteur, le terme $\theta \epsilon o \lambda o \gamma(a$ (qui apparaît ici pour la première fois dans la littérature occidentale) « ne désigne pas une science, mais le langage de ceux, poètes ou prosateurs, qui parlent des dieux $»^{7}$. D'autres auteurs, et non des moindres, partagent cet avis 8 . Par contre, pour un nombre plus limité de chercheurs, dont le plus récent est G. Naddaf", il faudrait comprendre ici $\theta \epsilon o \lambda o \gamma$ la dans le sens de «science, recherche, ou discours rationnel sur les entités divines». Pour notre part, nous pensons que le contexte du passage en question présente la $\theta \epsilon o \lambda o \gamma l a$ comme un synonyme de la $\mu v \theta$ o $\lambda o \gamma$ la des poètes. Dans cette optique, le premier avis cité se trouve le plus proche d'une interprétation plausible. Nous aimerions ajouter, toutefois, que l'élaboration des modèles théologiques par la recherche dialectique constituerait, quant à elle, une «théologie » dans le sens soutenu par la deuxième orientation.

Il est important de noter que les túmol $\pi \epsilon \rho \dot{~} \theta \epsilon o \lambda o \gamma \cos$ platoniciens figurent dans les livres II et III de la République, quand il est question de définir les qualités des futurs gardiens de la cité idéale et l'éducation qui peut le mieux les développer. Socrate estime qu'il faut établir certains règles afin d'obliger les poètes à ne rien clire de faux concernant les dieux et donc d'éviter de procurer aux jeunes des modèles de comportement nuisibles. Ces traits caractéristiques communs à toutes les divinités sont finalement définis par les interlocuteurs comme suit :

\footnotetext{
$4 \quad$ Platon, République II, 379a 7.

5 Cratyle, 400d 6 - 401a 7.

6 République II, 378e 4 - 379a 9.
}

7 R. BodéÜs, "La philosophie et les dieux du Phèdie", in L. Rossețtr (éd.), Understanding the Pbaedrus, Proceedings of the IInd Symposium Platonicum, Sankt Augustin, 1992, p. 247, n. 10. Cet auteur reprend la même idée dans Aristote et la théologie des vivants immortels, Paris/Montréal, 1992, p. 301-335.

8 Cf. V. Goldschmidt, "Theologia" [1949], in Questions platoniciennes, Paris, 1970, p. 141-172; G. VLastos, "Theology and Philosophy in Early Greek Thought" [1952], in Studies in Presocratic Philosophy I, London, 1970, p. 92-129; A. J. Festugrère, La révélation d'Hermès Trismégiste II : Le Dieu cosmique, Paris, 1949, p. 598-605.

9 G. NAdDaf, "Plato's Theologia Revisited", in Mêthexis 9 (1996), p. 5-18; cf. aussi W. JaEger, The Theology of the Early Greek Philosopbers, Oxford, 1947, p. 4-13. 
- Un dieu est bon et source des seuls biens. Même la punition que les dieux imposent à un homme pour ses méfaits est en accord avec la justice et vise à son amélioration. Elle est donc bienfaisante à long terme. L'origine de leurs maux, les hommes doivent la chercher ailleurs ${ }^{10}$.

- Les dieux ne peuvent pas commettre d'injustice, ni inciter quelqu'un d'autre à en commettre une ${ }^{11}$.

- Les dieux sont parfaits en ce qui concerne autant leur forme que leur vertu. Ils restent éternellement identiques à eux-mêmes, puisqu'ils réalisent déjà au plus haut

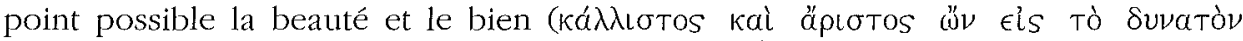

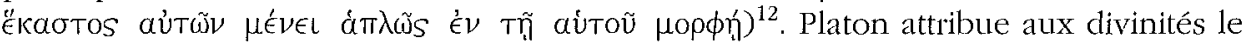
sommet de l'idéal traditionnel grec du kaloskagathos, qui porte sur l'ensemble d'un

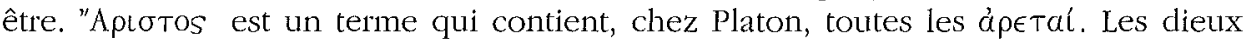
sont donc absolument sages, courageux, tempérants et justes. Platon est le premier penseur occidental qui insiste également sur la bonté absolue de la divinité.

Par conséquent, d'après le philosophe, les divinités ne peuvent que vivie en harmonie entre elles ${ }^{13}$. Elles ne cherchent jamais à tromper les hommes quand elles se mettent en communication avec eux; au contraire, elles leur transmettent la connaissance de la vérité stable et sûre - dont elles seules détiennent le secret - par des oracles, par des rêves ou par une inspiration divine. Les dieux ne peuvent non plus être attachés à des passions humaines, comme le désir charnel ${ }^{14}$ ou le gain pécuniaire $^{15}$, ni à des démonstrations d'émotions excessives de joie ${ }^{16}$ et de peine ${ }^{17}$. C'est ainsi que les divinités peuvent effectivement jouer le rôle de modèles de conduite éthique pour les hommes, un rôle que les croyances traditionnelles leur attribuaient couramment et que Platon accepte volontiers ${ }^{18}$.

\section{Quelques exceptions}

Le philosophe s'autorise donc à théologiser et à poser des règles rationnelles aux croyances humaines sur les dieux. En effet, nous constatons que Platon prend particulièrement soin de rester fidèle à ces principes quand il fait état des diverses divinités. Or, dans les dialogues apparaissent également quelques écarts - très rares, il est vrai - par rapport à ces lignes conductrices. Nous nous contentons ici de trois exemples représentatifs de ces cas exceptionnels, où Platon paraît oublier ses propres règles théologiques, afin de mieux comprendre leur raison d'être et leur impact sur la valeur que le philosophe accorde réellement aux typoi transgressés.

10 Platon, République II, 379d 1 - c 7

11 Ibid. II, 379e 3-380a 4 .

12 Ibid. II, 380d $1-381 \mathrm{c} 9$.

13 Ibid. II, 378 b8 - d 7.

14 Ibid. III, $390 \mathrm{~b} 6 \mathrm{sq}$.

15 Ibid. III, $408 \mathrm{~b} 6-\mathrm{c} 4$.

16 Ibid. III, 388e 5 - 389a 6.

17 Ibid. III, 387d 4-389a 1.

18 C'est donc pour des raisons ontologiques et éthiques, aussi bien que par souci pour la stabilité politique de la cité idéale (résultant seulement de l'acquisition de la vertu par les citoyens, et surtout par les gardiens), que Platon se tourne contre la liberté totale des poètes, une attitude qui a engendré de longs questionnements et des discussions toujours en cours. Malgré le grand intérêt de ce sujet, les limites du présent article nous obligent à ne pas nous y attarder. 
I. Dans les Lois $^{19}$, le législateur Athénien accuse les Crétois d'avoir inventé le mythe de Ganymède, le beau garçon enlevé par Zeus. Le « père des dieux » serait tombé amoureux du jeune mortel, devenu dans la suite l'échanson de l'Olympe. Ce serait donc pour présenter leurs propres penchants en matière de rapports amoureux comme une imitation de leur dieu protecteur que les Crétois auraient attribué cette attitude au dieu suprême. Pourtant, Socrate se réfère à ce même mythe dans le

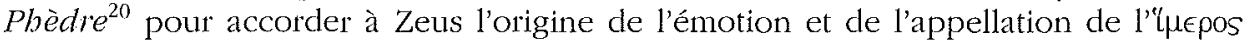
(une forme du désir). En le faisant, il transgresse aussi le principe de base selon lequel un dieu ne peut être la proie de ce genre de désirs.

Devant cette contradiction, on pourrait peut-être s'empresser de remarquer que le Pbèdre est antérieur à la République, alors que les Lois lui sont postérieures. La pensée de Platon n'aurait donc évolué dans la direction énoncée par les typoi theologias que vers la fin de sa période de maturité. Cependant, les indices recueillis dans les textes des premiers dialogues nous amènent à rejeter cette hypothèse. Platon présente une stabilité globale étonnante du début à la fin de son œuvre en ce qui concerne les traits caractéristiques généraux des divinités. Par contre, une autre remarque s'impose : les personnes qui se réfèrent au mythe de Ganymède ne sont pas les mêmes. Curieusement, c'est le législateur athénien qui s'accorde avec l'attitude de Socrate dans la République, alors que Socrate se trouve en contradiction - apparente, du moins - avec lui-même d'un dialogue à l'autre.

Il est vrai que, dans le Phèdre, la discussion porte sur la meilleure manière de vivre une relation amoureuse de type « homophile ${ }^{21}$. Zeus est présenté, dans le même dialogue, comme le guide des philosophes. Socrate veut surtout avancer ici que la manière "philosophique » d'envisager l'éros est la meilleure possible (le seul éros vrai), et inciter le jeune Phèdre à suivre celui-ci. Le philosophe a donc recours au même «stratagème » que les Crétois. La différence réside dans le fait que l'éros philosophique ne peut qu'être en accord avec le Bien et vise lui-même à l'éducation à la vraie vertu. Il serait donc celui qu'un dieu " philosophe ${ }^{22}$ pourrait ressentir.

II. Éros en tant que divinité présente l'exemple le plus flagrant de présentations controversées, en dépit du principe de la forme simple et unique des dieux.

Dans le Banquet, Pausanias et Éryximaque acceptent l'existence de deux Éros, l'un Ouranios (céleste, sage et respectable) et l'autre Pandémos (populaire et attaché aux plaisirs corporels). Ce dernier est condamné par Pausanias, alors qu'Éryximaque conseille de l'honorer avec modération. Pausanias avance de surcroît l'existence de deux Aphrodites correspondantes ${ }^{23}$. Socrate démentira dans la suite ces positions, aussi bien que les diverses versions de la généalogie et des œuvres d'Éros proposées

19 Platon, Lois 1, 636c 7 - e 3.

20 Pbèdre, $255 b 7 \mathrm{sq}$.

21 C'est le terme actuellement utilisé pour désigner l'éros éducatif entre un homme mûr et un éphèbe, habitude sociale particulière de l'antiquité, à ne pas confondre avec les rapports homosexuels entre adultes, ni avec la perversion de la pédophilie. Voir aussi l'article éclairant de L. Brisson, "Le Banquet de Platon comme document sur les comportements sexuels et leurs représentations sociales", in L.G. TrN (dir), Homosexualités: expression/répression, avec la collaboration de G. Pastre, Paris, 2000, p. 51-62.

22 Dans le Pbèdre, Zeus est présenté comme le dieu protecteur des philosophes, lesquels

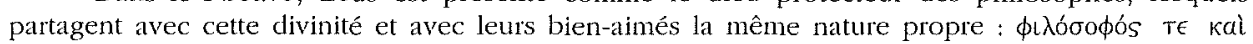

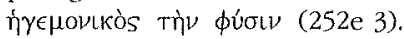

23 Platon, Banquet, 180d 3 - e 2; 184b 5-185c 3 (Pausanias) et 187a 1 - e 3 (Éryximaque). 
par les autres convives. En rapportant l'enseignement de Diotime, le philosophe présente à son tour Éros comme « un grand daimôn », appartenant dès lors à la catégorie du metaxu, fils de Poros et de Pénia, conçu le jour de la naissance d'Aphrodite ${ }^{24}$.

Pourtant, ce même Socrate déclare, dans le Phèdre, qu'Éros est « un grand dieu », fils d'Aphrodite ${ }^{25}$. Qu'en est-il ? $^{26}$ En fait, dans le Banquet, Platon met en évidence la grande diversité de croyances sur Éros. La raison de ces divergences peut résider non seulement dans le fait que les Grecs jouissaient d'une grande liberté dans le domaine de la mythologie, mais aussi dans la nature même d'Éros. Cette divinité se trouve souvent confondue avec la fonction psychique homonyme; chacun projette, dès lors, sur le dieu ses propres expériences amoureuses.

Si cela peut justifier les controverses parmi les différentes personnes qui profèrent les mythes sur Éros, et qui ne représentent pas nécessairement l'avis de Platon, le cas de Socrate pose encore un problème. Qui plus est, le maître de Platon tient dans les dialogues le rôle du philosophe paradigmatique; il peut être considéré comme le représentant par excellence des conceptions que l'auteur veut avancer. Les difficultés peuvent être en partie justifiées par la fonction des mentions d'Éros dans chacun de ses dialogues. Dans le Banquet, la qualification de cette divinité comme un « grand daimôn » philosophe permet de définir le philosophe aussi comme un intermédiaire qui assure la communication entre les deux niveaux ontologiques : celui de la divinité, où résident les dieux et les «vérités divines » (dont, par exemple, l'Idée du Beau), et celui des hommes. Dans le Phèdre, c'est la nature divine d'Éros qui est soulignée, car Platon désire surtout opposer l'Éros philosophique (divin) à toute autre manifestation de désir amoureux qui ne vise pas au Bien.

III. Dans le Cratyle, Socrate présente Hermès comme l'inventeur du logos. Curieusement, il n'attribue pas au dieu seulement la parole qui reflète la vérité, mais aussi celle qui peut tromper ${ }^{27}$, en faisant référencee à l'esprit rusé d'Hermès, connu par les conceptions courantes. Or, dans la suite, Socrate met en parallèle les deux fils d'Hermès, Pan et le logos. La double nature nature de Pan (mi-divine, mi-animale) sert à ilustrer les deux aspects de la parole : le vrai (résidant auprès des dieux) et le faux (qui circule parmi les hommes ${ }^{28}$. Dans ce dialogue, Platon cherche à établir cette distinction entre le logos vrai de la philosophie représentant la nature des êtres et le logos faux des sophistes, qui se limite au niveau du vraisembiable. Si l'on s'en tenait à la perfection de la nature divine d'Hermès, ce dieu ne saurait tromper les hommes par ses inventions et donc tout logos serait vrai. Notons que Socrate reprend le même rapport entre Hermès, Pan et le logos dans le Phèdre, dont la deuxième partie est consacrée à l'art de la parole et à la distinction entre le logos philosophique (vrai) et celui des rhéteurs (vraisemblable) ${ }^{29}$.

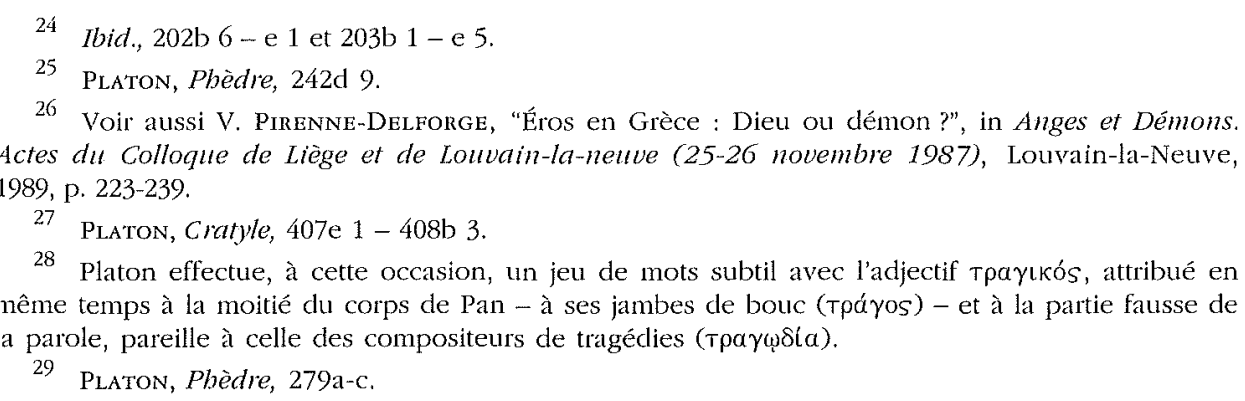




\section{Tentatives d'interprétation}

Nous avons vu que, dans les cas examinés, les « écarts » de Platon par rapport à ses propres typoi theologias peuvent être dus à la forme dialectique adoptée par le philosophe, où les différentes personnages énoncent des avis que l'auteur n'épouse pas nécessairement. D'autre part, Socrate lui-même, qui représente par excellence les opinions du philosophe, peut tenir des propos opposés aux typoi theologias, voire même contradictoires entre eux, si les besoins de l'argumentation du dialogue spécifique l'exigent. Platon paraît donc adapter l'image des divinités traditionnelles aux objectifs de son texte philosophique, qui vise lui-même à la recherche de la vérité et à l'éducation à la vertu. Cette constatation suscite des questions multiples, surtout si l'on prend en consicération certains passages où Platon manifeste également sa distance par rapport à la vérité des mythes sur les dieux, diffusés par le législateurphilosophe.

Dans la République, à l'occasion des récits sur l'attitude violente de Kronos envers son père, Ouranos ${ }^{30}$, Socrate insiste sur le fait que, même si ces mythes étaient vrais, il ne faudrait les diffuser que dans un petit cercle d'initiés et ne pas les laisser circuler dans la cité, car ceux-ci constituent de mauvais exemples pour les jeunes. D'autre part, Socrate indique qu'il faut cultiver certaines croyances bénéfiques chez les citoyens en créant des mythes approptiés, comme celui de Gé, la Terre, qui serait la mère originelle de tous les hommes du pays ${ }^{31}$. Platon justifie donc l'éventualité à la fois de «pieux mensonges » et de censure de la part du philosophe-législateur en matière de mythologie.

La réflexion sur les représentations des dieux conformément aux typoi peri theologias figure aussi dans le cadre du rôle du philosophe en tant qu'éducateur des citoyens à la vertu dont l'acquisition garantit, selon Platon, le bonheur personnel et civique. Devrait-on dès lors conclure, comme P. Boyancé ${ }^{32}$, que la théologie platonicienne est dirigée par une finalité pratique, éthique, politique ou éducative? Qui plus est, si l'on prend en considération l'« agnosticisme » exprimé à plusieurs reprises par Platon concernant l'incapacité naturelle des hommes à atteindre une connaissance certaine sur les dieux ${ }^{33}$, peut-on prendre au sérieux tout logos platonicien qui se réfère à la divinité ?

Certains chercheurs, comme F. Solmsen ${ }^{34}$, ont utilisé cet argument pour remettre entièrement en question la croyance ou même le respect de Platon face à la religion traditionnelle et à ses divinités. Cependant, R. Bodéü ${ }^{35}$ a soutenu fort judicieusement que la définition des typoi theologias offre une solution au problème épistémologique de la possibilité de la connaissance sur les dieux. En posant, par la méthode dialectique, l'Idée des dieux, Platon rend la divinité accessible à notre intellect. Cette définition est effectuée par Socrate lui-même, qui reste toujours un philosophe à la recherche de la vérité. Si une certaine flexibilité est permise en ce qui concerne la diffusion de quelques mythes dans le grand public (et encore, la manière dont Platon

30 République II, 367e $6-378 \mathrm{~b} 4$.

31 Ibid. III, 414d 2 - 415a 1.

32 P. Boyancé, Le culte des Muses chez les pbilosophes grecs, Paris, 1972, p. 159.

33 Cf. Platon, Sophiste, 218c-d; Politique, 277d; Criton, 107b; République II, 368d; Lois II, 644e; Cratyle, 400d-401a; Pbilèbe, 12a-c.

34 Voir F. Solmsen, Plato's Theology, Ithaca/New York, 1942, p. 118-119; 122, n. 52.

35 Voir Bodéüs, l.c. (n. 7), p. 246-248. 
en parle dévoile sa susceptibilité face à la véracité de ces histoires), le philosophe a atteint au préalable la représentation de la divinité la plus fidèle possible. De surcroît, l'accès du philosophe à la vérité n'est pas réalisé seulement par la voie de ses propres fonctions rationelles. À diverses reprises, Socrate prétend aussi à une inspiration divine $^{36}$ qui garantit traditionnelement l'accès à la vérité à un niveau sur-humain. Plusieuirs platonisants, comme par exemple A. Diès ${ }^{37}$ et V. Goldschmidt ${ }^{38}$, ont même vu une identification entre l'exercice de la dialectique, qui est une démarche rationnelle, et l'attitude « religieuse » de Platon, à connotations mystiques (aspiration à la contemplation des Idées).

Nous ne partageons pas ces avis, mais nous devons accepter que, pour Platon, le philosophe se présente comme étant clairement en mesure d'avoir une connaissance de la divinité « autant qu'il soit possible à l'homme », et ce, autant par les moyens traditionnels d'une « inspiration divine » passive, que par une nouvelle voie, active et consciente, celle de la démarche dialectique. En effet, Platon réalise une distinction entre le niveau proprement épistémologique de la connaissance de l'Idée de la divinité par le philosophe grâce à la dialectique et celui de l'application de cette connaissance aux croyances publiques, où c'est le bien politique qui prédomine (étant donné que ces croyances forment l'éthique des citoyens). L'inconstance apparente de certaines croyances énoncées dans les dialogues s'intègre, à notre avis, dans ce même cadre de finalité éducative qu'assument les textes platoniciens. Néanmoins, on ne peut pas parler d'une opposition essentielle entre les deux niveaux de représentations de la divinité, car les mêmes règles rationnelles sont en principe valables dans les deux cas.

\section{Épilogue}

Pour Platon il y a largement coïncidence entre le Vrai, le Bien et le Beau. Ses principes théologiques répondent à la fois à des critères logiques, religieux, éthiques, politiques et éducatifs, comme l'a remarqué D. Babut ${ }^{39}$. La manière dont ces principes seront appliqués dans la représentation publique des divinités traditionnelles reste, pourtant, prioritairement en accord avec le Bien. Le rationalisme du philosophe, quand il est question de croyances publiques dont l'impact politique est considérable, s'aligne et, si besoin est, se soumet à son humanisme profond. En cela, Platon imite lui-même le trait le plus original dont il a doté les dieux traditionnels: la bonté envers les hommes. Notons que, pour souligner le rapport du philosophe avec les divinités comme paradigmes, Platon n'hésite pas à qualifier certains dieux de

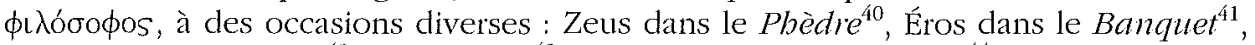
Athéna dans le Timée $e^{42}$ et le Critias $^{43}$, Héphaïstos dans le Critias $^{44}$ et Hadès dans le

36 Voir, par exemple, Platon, Phédon, 60e 4-61b 7; 84e 3-85b 7; Phèdre, 238c 5-d 5.

37 A. Diès, Autour de Platon. Essais de critique et d'bistoire II : Les dialogues - Esquisses doctrinales, Paris, 1927 (Bibliotbèque des Archives de Pbilosopbie), p. 592.

38 V. Goldschmidt, La Religion de Plalon, Paris, 1949, p. 27.

39 D. BaBuT, La religion des philosophes grecs, Paris, 1974, p. 94.

40 Platon, Phèdre, 253e 1-3.

41 Banquet, 204b-c.

42 Timée, $24 \mathrm{c} 4-\mathrm{d} 3$.

43 Critias, 113a 1 - b 6.

44 Ibid., $113 \mathrm{a} 1$ - b 6 . 
Cratyle $e^{45}$. Dans les Lois $^{46}$, il est également question de la Muse philosophique. Par cette qualification Platon ne remet pas en question la oo vent aimer particulièrement la sagesse, tout en la possédant ${ }^{47}$, ou ils peuvent pratiquer déjà la dialectique, comme le philosophe tente de le faire tout au long de son existence, qui est si souvent qualifiée de ó l'intellect, une nouvelle activité humaine, qui se place dès lors sous les auspices de la divinité, ce qui garantit non seulement sa «légitimité », mais aussi son caractère bienfaisant.

Nous croyons que ce dernier exemple de «transgression » apparente des règles théologiques amène une preuve supplémentaire de l'attitude complexe de Platon. En effet, le philosophe introduit, d'une part, des éléments rationnels propres à la philosophie dans la définition des croyances religieuses et, d'autre part, soutient l'exercice de la dialectique avec ces mêmes croyances. Ce « rapport dialectique » entre rationalité et religion, dont nous n'avons examiné ici que quelques exemples, paraît se soumettre aux exigences de l'incontournable souveraineté de l'Idée du Bien.

Centre universitaire de Luxembourg / Université de Liège

Aikaterini LEFKA

Bd. d'Avioy, $77 / 111$

B - 4000 Lì̀GE

45 Platon, Cratyle, $403 \mathrm{~d} 7-404 \mathrm{a} 6$.

46 Lois VI, 499c $7-\mathrm{d} 6$.

47 pour Platon, la sagesse des dieux est maintenue par la contemplation régulière des Idées (Pbèdre, 246e $4 \mathrm{sq}$.). Il s'agit d'une conception originale du philosophe qui met en rapport les données connues des croyances traditionnelles avec sa propre métaphysique et son épistémologie. 